\title{
Analysis of Gas Permeability Characteristics of Poly(Lactic Acid)/Poly(Butylene Succinate) Nanocomposites
}

\author{
Amita Bhatia, ${ }^{1}$ Rahul K. Gupta, ${ }^{1}$ Sati N. Bhattacharya, ${ }^{1}$ and Hyoung Jin Choi $^{2}$ \\ ${ }^{1}$ Rheology and Materials Processing Centre, School of Civil, Environmental and Chemical Engineering, \\ RMIT University, Melbourne, VIC 3001, Australia \\ ${ }^{2}$ Department of Polymer Science and Engineering, Inha University, Incheon 402-751, Republic of Korea
}

Correspondence should be addressed to Rahul K. Gupta, rahul.gupta@rmit.edu.au and Hyoung Jin Choi, hjchoi@inha.ac.kr

Received 29 June 2012; Revised 13 September 2012; Accepted 13 September 2012

Academic Editor: Suprakas Sinha Ray

Copyright () 2012 Amita Bhatia et al. This is an open access article distributed under the Creative Commons Attribution License, which permits unrestricted use, distribution, and reproduction in any medium, provided the original work is properly cited.

\begin{abstract}
Gas permeability and morphological properties of nanocomposites prepared by the mixing of poly(lactic acid) (PLA), poly(butylene succinate) (PBS), and clay was investigated. While the composition of PLA and PBS polymers was fixed as $80 \%$ and $20 \%$ by weight, respectively, for all the nanocomposites, clay contents varied from 1 to $10 \mathrm{wt} \%$. From the morphological studies using both wide angle X-ray diffraction and transmission electron microscopy, the nanocomposite having $1 \mathrm{wt} \%$ of clay was considered to have a mixed morphology of intercalated and delaminated structure, while some clusters or agglomerated particles were detected for nanocomposites having 3 and more than $3 \mathrm{wt} \%$ of clay content. However, the average particle size of the dispersed PBS phase was reduced significantly from $7 \mu \mathrm{m}$ to $30-40 \mathrm{~nm}$ with the addition of clay in the blend. The oxygen barrier property was improved significantly as compared to the water vapor. A model based on gas barrier property was used for the validation of the oxygen relative permeabilities of PLA/PBS/clay nanocomposites. PLA/PBS/clay nanocomposites validated the Bharadwaj model up to $3 \mathrm{wt} \%$ of clay contents only, while for nanocomposites of higher clay contents the Bharadwaj model was invalid due to the clusters and agglomerates formed.
\end{abstract}

\section{Introduction}

Polymer/organically modified layered silicate nanocomposites have attracted much attention in the past few decades because of their demonstrated significant enhancement of a large number of the properties including barrier, thermal, mechanical, rheological, flammability resistance, and other physical properties relative to an unmodified silicate or neat polymer resin. These improvements are mainly achieved at lower silicate loadings $(\leq 5 \mathrm{wt} \%)$, which result in a high aspect ratio along with a large surface area. The concentration of silicate loading required for the preparation of nanocomposites is a fraction of what is typically required with conventional composites. Moreover, the improvement in these nanocomposites is matchable or higher than those for the conventional composites regarding the filler content. Till now, various nanocomposites have been developed and are being developed using layered silicate clay minerals as a reinforcing phase due to its easy and abundant availability, low cost, and, more importantly, environmental friendliness [1].

The rapid growth of the use of plastics in our daily life has become an important issue due to the resulting global environmental problems. Generally, polyethylene, polypropylene, polystyrene, poly(vinyl chloride), and so forth are the most produced, used, and then discarded in the environment. Especially, their packaging materials end up as dangerous, undegradable wastes. Due to the unavailability of satisfactory landfill sites, production of large amounts of carbon dioxide and toxic gases during incineration, which again contribute to the environmental pollution, and the more expensive and reduced quality of recycled plastic products, there has been a drive to concentrate on 
biodegradable polymeric materials. Keeping these reasons in mind, the development of biodegradable materials with superior properties has become a challenging and interesting subject for researchers, scientists, and engineers throughout the world today. It is expected that, like other polymer nanocomposites, biodegradable polymer-nanocomposites will also demonstrate great enhancements in physical and material properties. Therefore, these biodegradable nanocomposites may become a possible solution for the global environmental problem caused by plastic wastes.

Various researchers $[2,3]$ have studied biodegradable polymers which are derived from renewable and petroleum resources; these include poly(L-lactide) (PLA), poly(butylene succinate) (PBS), poly(butylene adipate terephthalate), poly(ethylene terephthalate succinate), and $\operatorname{poly}(\varepsilon-$ caprolactone). The PLA, which is produced from a feedstock of corn rather than petroleum, has recently been used for packaging material. Packaging material for food should provide sufficient barriers against water vapour to prevent the food degradation, against atmospheric gases to prevent the oxidation of the food material, and against volatile organic compounds to preserve the aromas and the flavors of the food [4]. In food packaging, mass transfer both from the food to the polymer and vice-versa is responsible for the contamination of food and also causes degradation of the packaging material. Since the PLA is produced from a lactide monomer coming from a lactic acid which is a nontoxic substance already found in the human body, food contamination can be neglected when the PLA is used as a packaging material. Therefore, it can be assumed that the determination of permeability of oxygen, water vapour, and aromas in PLA used for food packaging material is an important parameter.

PLA is a linear thermoplastic polyester. It has good mechanical properties, thermal plasticity, and good biocompatibility and it can be easily fabricated. However, the cost associated with the ring-opening polymerization of lactide monomer and brittleness of the PLA limit the commercial use of PLA polymer. Therefore, blending of PLA with another biodegradable polyester polymer may be necessary such that the resultant blend would overcome the limitations of PLA achieving good qualities by the addition of another polymer and also reduced the final cost.

In this study, the polymer selected to blend with PLA was PBS which is one of the most promising polymers in the family of synthetic biodegradable polyesters. PBS, chemically synthesized by the polycondensation of 1, 4-butanediol with succinic acid [5], has high flexibility, excellent impact strength, and thermal and chemical resistance [6]. Excellent processability of the PBS might make processability PLA better. Among various physical characteristics, gas permeability properties of the nanocomposites are mainly focused and then correlated with their morphological properties from both XRD and TEM.

\section{Background of Gas Barrier Modeling}

Theoretical models have been developed for predicting the properties of composite materials based on the properties of the pure components and the morphology of the composite. The general objective of such theories has been to predict the performance of the composite for a given set of components [7]. These theories enable a simple route for evaluation of the individual contribution of the component's properties, such as matrix type, volume fraction, filler aspect ratio, filler orientation, and filler distribution. Various studies $[8,9]$ have shown that aspect ratio and particle distribution in the polymer matrix are important factors for predicting the properties of polymer nanocomposites; suitable values help in achieving high enhancement in properties of nanocomposites.

Enhancements of various properties are well known in polymer-silicate nanocomposites; their main dependence is on the distribution of the silicate layers in the polymer matrices. However, the main factors behind the improvement in gas barrier properties are not yet fully understood. As clays are crystalline materials, they are believed to increase the barrier properties by creating a maze or "tortuous path" that restricts the progress of the gas molecules to pass through the polymer matrices [10]. Note that the idea of "tortuosity" has been already introduced by Michaels and Parker [11] accounting for the impedance to flow offered by the irregular intercrystallite passages of polyethylene.

Nielsen [10] developed a theory for diffusion through filled polymer systems. According to this theory, if the fillers are impenetrable to a diffusing gas or liquid molecule, then the diffusing molecule must go around the filler particles. He utilized the tortuosity factor $(\tau)$ which is defined as the ratio of the actual distance $\left(d^{\prime}\right)$ that a penetrant must travel to the shortest distance $(d)$ that it would have travelled in the absence of the layered silicate. Nielson added that thin, plate-like fillers, having a large aspect ratio $(L / 2 W)$ (where $L$ is the length of the filler particle and $W$ is the width of the filler plates), can dramatically reduce the permeability only if the particles are oriented in such a way that their flat surfaces are parallel to the surfaces of the film. Nielsen also stressed that incomplete filler dispersion, voids, and only partial orientation of the particles should result in a reverse affect on permeability. The tortuosity factor $(\tau)$ is expressed as

$$
\tau=\frac{d^{\prime}}{d}=1+\frac{L}{2 W} \phi_{s},
$$

where $\phi_{s}$ is the volume fraction of the sheets.

The effect of tortuosity on the permeability can be expressed as

$$
\frac{P_{s}}{P_{p}}=\frac{1-\phi_{s}}{1+L / 2 W} \phi_{s}
$$

where $P_{s}$ and $P_{p}$ represent the permeabilities of the nanocomposite and neat polymer, respectively.

Bharadwaj [12] invoked a simple model, developed earlier by Nielsen, correlating the sheet length, concentration, relative orientation, and state of aggregation of the filler in the polymer matrix. This model gave further guidance in the design of better barrier materials for nanocomposites. Bharadwaj [12] predicted, from (2), that the relative 
permeability $\left(P_{s} / P_{p}\right)$ is a function of the silicate sheet length. The relative permeability of $\mathrm{H}_{2} \mathrm{O}$ in a polyimidemontmorillonite nanocomposite reproduced the proposed concentration dependence according to (2) [13], showing a progressive decrease in relative permeabilities as a function of increasing concentration and sheet length. Extremely large length-to-thickness ratio of the silicate sheets (30 to $\sim 2000 \mathrm{~nm}$ in length, $1 \mathrm{~nm}$ thick) showed a significant increase in tortuosity. It is interesting to note that no significant reduction in the relative permeability was noticed, regardless of the clay sheet length, beyond $\Phi_{s}=0.05$. This is also in agreement with experimental observations [13-18].

Bharadwaj modified the tortuosity factor to include the orientational order $(S)$, writing the relative permeability as follows:

$$
\frac{P_{s}}{P_{p}}=\frac{1-\phi_{s}}{1+(L / 2 W) \phi_{s}(2 / 3)(S+1 / 2)} .
$$

The above expression reduces to (2) when $S=1$ (planar arrangement, i.e., maximum tortuosity) and provides approximately the permeability of the neat polymer when $S=-1 / 2$ (orthogonal arrangement). Further, Bharadwaj concluded that incorporating sheets with $L>500 \mathrm{~nm}$ with random orientation was more beneficial for the barrier properties than the case where the sheets were aligned perpendicular to the diffusing path.

Exfoliation appears to be the critical factor in determining the maximum performance of polymer nanocomposites for barrier applications. A significant decrease in the permeability of $\mathrm{O}_{2}$ gas through the near exfoliated PLAnanocomposite relative to the neat polymer (PLA) was clearly observed [8]. Nielsen [10] explained that at $L=$ $1000 \mathrm{~nm}$ the relative permeability was $\sim 0.05$ for $W=1 \mathrm{~nm}$ and 0.2 for $W=7 \mathrm{~nm}$. While for $L=500 \mathrm{~nm}$, relative permeability changed from 0.1 to 0.3 , respectively, for $W$ $=1$ to $7 \mathrm{~nm}$, thus underscoring the need for complete delamination of the silicate sheets.

Messersmith and Giannelis [19] observed a dramatic reduction in water permeability through composite films of poly(epsilon caprolactone)/organically modified mica-type silicate containing modest amounts of silicate. This reduction was due to the dispersion of impermeable, high aspect ratio silicate layers within the polymer matrix. On the other hand, Cava et al. [20] compared the barrier properties of biodegradable plastics and their nanocomposites with PET for food packaging applications. Biodegradable materials showed higher oxygen permeability compared to PET at $21^{\circ} \mathrm{C}$ and $40 \%$ relative humidity; however, their nanocomposites demonstrated marginally lower permeability to the test gas than the neat polymer.

Both the Nielsen [10] and Bharadwaj [12] models are based on arguments related to the tortuosity factor. Further, equations related to the permeability and tortuosity were developed to understand the diffusion of small molecules in conventional composites; however, they do extremely well in predicting and relating the experimental results for the relative permeability in polymer/layered silicate nanocomposites. The main assumption of the above models is that the sheets are oriented, such that the sheet normal is coincident with the direction of diffusion $(S=1)$. Clearly, this arrangement results in the highest tortuosity. Any deviation from this arrangement would lead to deterioration in the barrier properties. Also, a sheet-like morphology $(W=1)$ is particularly efficient at maximizing the path length of the penetration due to the large length-to-width ratio compared to spheres or cubes.

The purpose of this research was to better understand the influence of clay concentration on the gas barrier properties and morphology of biodegradable nanocomposites of PLA/PBS/C30BX (an experimental grade related to Cloisite 30B) nanocomposites having a fixed composition of PLA and PBS, at $80 \mathrm{wt} \%$ and $2 \mathrm{wt} \%$, respectively, with various clay loadings. Various aspects of selected morphological, oxygen, and water vapour permeability properties of the nanocomposites were compared to those of the polymer blend without clay. In particular, this study mainly aimed to obtain an extensive understanding of the gas barrier properties of biodegradable polymer nanocomposites using the Bharadwaj model based on detailed morphological analysis from TEM images. Comparison of oxygen relative permeabilities obtained through experimental investigations with relative permeabilities calculated from the Bharadwaj model was done. Bharadwaj model predictions were also discussed in terms of the morphological structure of the nanocomposites reanalyzed from TEM images reported [21].

\section{Experimental}

3.1. Materials. Both biodegradable poly(lactic acid) (4032Dgrade, Nature Works, Cargill Dow LLC) and poly(butylene succinate) (PBS) (grade G4460, Ire Chem. Co., Republic of Korea) were used in this study for blending. Densities of PLA and PBS were $1.240 \mathrm{~g} / \mathrm{cm}^{3}$ and $1.230 \mathrm{~g} / \mathrm{cm}^{3}$, respectively. The PLA had crystalline melting point range between 150 and $170^{\circ} \mathrm{C}$, while PBS had a melting range between 95 and $114.7^{\circ} \mathrm{C}$. Furthermore, an experimental grade of Cloisite 30B (SCPX 3016, Southern Clay Products (SCP) Inc., USA) organoclay, indexed as C30BX, was used. The experimental grade clay is known to provide better dispersion in biodegradable polymers, such as PLA and PBS matrices, as compared to the commercially used C30B. This clay has basically the same chemical treatment as $\mathrm{C} 30 \mathrm{~B}$ but it was achieved via a different mechanism. The C30BX, was produced by a cation exchange reaction whereby natural MMT $\left(\mathrm{Na}^{+}\right.$-MMT) was chemically modified with bis-(2hyroxyethy) methyl (hydrogenated tallow alkyl) ammonium cations $\left.\left(\mathrm{C}_{18} \mathrm{H}_{37}\right)-\mathrm{N}^{+}\left(\mathrm{C}_{2} \mathrm{H}_{4} \mathrm{OH}\right)_{2} \mathrm{CH}_{3}\right)$.

3.2. Preparation of PLA/PBS/Clay Nanocomposites. Before processing through an extruder, the pellets of both PLA and PBS were first dried in a vacuum oven at a temperature of $50^{\circ} \mathrm{C}$ for $48 \mathrm{~h}$ to remove moisture. Drying of these polymers is very essential as moisture in these polymers induces in hydrolytic chain scission during the processing at high temperature. All the PLA/PBS/clay nancomposites were prepared by melt compounding using a Brabender twinscrew extruder at temperature $180^{\circ} \mathrm{C}$ and screw speed at 
$40 \mathrm{rpm}$. All the nanocomposites were passed twice through the extruder to achieve better and homogeneous mixing of all the compositions. The composition of the binary blends of the PLA/PBS was kept fixed at 80/20 (wt\%/wt\%) for all the nanocomposites. Nanocomposites of various clay loadings, $1,3,5,7$, and 10 by wt $\%$, were prepared for the investigations in this study.

All the components were premixed in a plastic bag by tumbling well and were fed simultaneously into the extruder to maintain the overall consistency. The extruded nanocomposites were pelletized and dried again at $50^{\circ} \mathrm{C}$ in a vacuum oven over night. For the determination of oxygen and water vapour transmission rates, film samples of approximately $0.3 \mathrm{~mm}$ thickness, obtained by compression molding at $200^{\circ} \mathrm{C}$ and pressure of $40 \mathrm{kN}$ for 5 minutes, were used and the samples were then cooled under pressure $(20 \mathrm{MPa})$ for $2-3 \mathrm{~min}$. All these samples were used for morphological and gas barrier property evaluations.

3.3. Analysis of PLA/PBS/Clay Nanocomposites. To examine morphology of the nanocomposites, wide angle X-ray diffraction (WAXD) (Philips PW1847 X-ray diffractometer) was carried out with a reflection geometry and $\mathrm{CuK}_{\alpha}$ radiation (wavelength $\lambda=0.154 \mathrm{~nm}$ ) operated at $40 \mathrm{kV}$ and $30 \mathrm{~mA}$ within the range of scattering angles $(2 \theta)$ from 1 to $10^{\circ}$. To investigate the internal structure and dispersion state of the nanocomposites, ultrathin sections (less than $100 \mathrm{~nm}$ ) were microtomed using a Super NOVA instrument with diamond knife. Consequently, the ultrathin samples were investigated using a transmission electron microscopy (TEM) (Philips CM200) operated at 100-kV accelerating voltage [22].

For gas permeability study, at first oxygen permeability was examined with a MOCON Ox-Tran 2/21 instrument (Modern Controls Inc., USA) according to ASTM D3985 standard as previously described [23]. The values of oxygen permeability were obtained at $23^{\circ} \mathrm{C}$ and $0 \% \mathrm{RH}$, with the exposed area and thickness of $50 \mathrm{~cm}^{2}$ and $0.3 \mathrm{~mm}$, respectively. A mixture of $97 \% \mathrm{~N}_{2}$ with $3 \% \mathrm{H}_{2}$ was used as a carrier gas and $99.9 \%$ oxygen was used as the test gas. The permeability of Mylar (polyester) film (MOCON manufacturer) was used to calibrate the instrument, in which the permeability of this Mylar film was already known. The film samples were placed in two test (permeability) cells. After installing the samples, a continuous flow of gas was maintained on both sides of the barrier material. Firstly, nitrogen gas was passed through both surfaces to remove any oxygen in the sample. The nitrogen on one side was then replaced by oxygen, and the nitrogen flow on the other side then swept the surface to extract any oxygen that diffused through the material. The diffusing oxygen was measured by a coulometric sensor detector that is sensitive only to oxygen. For the completion of one test after films were loaded in the test cells, it takes 12 to $16 \mathrm{~h}$. Before each measurement, the samples were kept at $50^{\circ} \mathrm{C}$ in a vacuum oven for an overnight, at least four samples of each film for their analysis.

The water vapour transmission rate (WVTR) of each PLA/PBS/C30BX nanocomposite sample was measured using a Modern Controls Inc. (MOCON) Permatran-W1 (USA), according to ASTM standard F 1249 at $100 \%$ relative humidity $(\mathrm{RH})$ and $37.8^{\circ} \mathrm{C}$ temperature. The exposure area for the WVTR measurements was $50 \mathrm{~cm}^{2}$ and thickness of the film sample was $0.3 \mathrm{~mm}$, as above. Water vapour transmission rate through the plastic film was measured using a modulated infrared sensor. Water vapour transmission rates can be evaluated for the films by using other solutions, that is, sodium chloride, sodium acetate, and magnesium chloride giving different relative humidity $(\mathrm{RH})$. In this study, very pure water $(100 \% \mathrm{RH})$ was used as the samples did not absorb much water. Calibration of this instrument was also done using a Mylar (polyester) film of 5 mil thickness supplied by MOCON, which has a WVTR of $4.55 \mathrm{~g} / \mathrm{m}^{2}$. day at $37.8^{\circ} \mathrm{C}$ and $100 \% \mathrm{RH}$. Test films mounted in the diffusion cell were initially exposed to a continuous flow of air across the upper side, while the bottom side was exposed to water vapour from the moistened pad (reverse osmosis water) in the humid cavity. This is called conditioning which took one night. Conditioning of the sample is required to allow the establishment of an equilibrium transmission rate. The flow rate of the air during the test was set to $60 \mathrm{~cm}^{3} / \mathrm{min}$. After conditioning, actual water vapour transmission (WVTR) was noted, usually after 12-16 hours. An average of five values is reported in this work after normalizing the unit values according to the thickness of the sample.

\section{Results and Discussion}

The characteristic peak of pristine C30BX in WAXD patterns occurred at the diffraction angle $4.96^{\circ}$. By using Bragg's law, the $d$-spacing, also called the basal spacing, was calculated as $1.78 \mathrm{~nm}$ for C30BX [23]. This is in close agreement with that of commercially available $\mathrm{C} 30 \mathrm{~B}$ organoclay (i.e., $1.75 \mathrm{~nm})$. The WAXD patterns of the PLA/PBS/C30BX nanocomposites from our previous work (Figure 1 [23]) demonstrated that the nanocomposite having $1 \mathrm{wt} \%$ of the C30BX did not show any diffraction peak in the range used, probably due to its low clay content. The nanocomposite of $3 \mathrm{wt} \%$ of C30BX exhibited a broad and extremely weak reflection at approximately $2^{\circ}$ diffraction angle. Moreover, it also showed a slightly visible peak at approximately $6^{\circ}$ diffraction angle. However, it is not sufficiently clear to be called a peak. For 5, 7, and $10 \mathrm{wt} \%$ of clay nanocomposites the peak shifted to lower angles at $2.3^{\circ}, 2.2^{\circ}$, and $2.2^{\circ}$, respectively. This indicated that the polymer chains were intercalated into the C30BX layers. The peak for the $5 \mathrm{wt} \%$ sample was relatively broad. Compared to the characteristic peak of neat C30BX the gave an interlayer spacing $\left(d_{001}\right)$ of $1.78 \mathrm{~nm}$, the interlayer $d$-spacing increased to $3.84 \mathrm{~nm}$, $4.01 \mathrm{~nm}$, and $4.01 \mathrm{~nm}$ in nanocomposites having 5,7 , and $10 \mathrm{wt} \%$ of C30BX concentration, respectively. Shifting of the peak occurred due to the intercalation of the polymer chains into the clay galleries, while broadening of the peak in 3 and $5 \mathrm{wt} \%$ of polymer/clay nanocomposite indicated a distribution of intercalated layer spacings. Krikorian and Pochan [24] revealed that the broadening of the peak can also occur due to the partial disruption of parallel stacking 


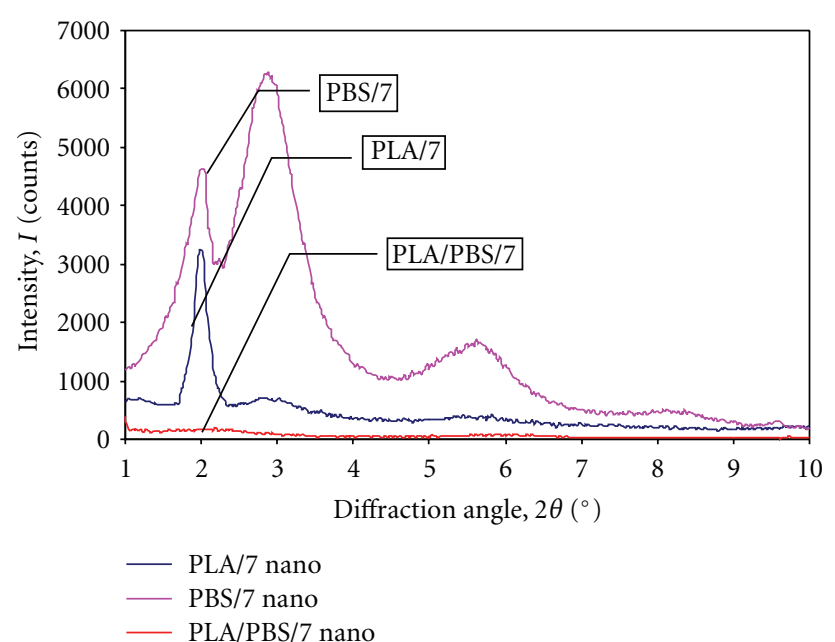

Figure 1: WAXD profiles of PLA/PBS/C30BX, PLA/C30BX, and $\mathrm{PBS} / \mathrm{C} 30 \mathrm{BX}$ nanocomposites. The content of C30BX was $7 \mathrm{wt} \%$ in all these nanocomposites.

or layer registry of the pristine organoclay, which means the existence of some delamination of clay platelets. Therefore, it can be concluded that a mixture of delaminated and intercalated structure was observed. On the other hand, nanocomposites with 7 and $10 \mathrm{wt} \%$ of clay clearly demonstrated predominantly intercalated structure, in which the intensity of these intercalated clay peaks gradually increased with the addition of the clay, due to more diffracted X-rays from the parallel assembly of the additional intercalated clay [24]. Interestingly, it was noticed that for the 3, 5, 7, and $10 \mathrm{wt} \%$ clay, the main diffraction peaks were accompanied by the appearance of another peak at $2 \theta$ of approximately $6^{\circ}$, at the same location with the same basal spacing for each nanocomposite. Note that an additional small peak at a diffraction angle $(2 \theta)$ below $10^{\circ}$ for various PLA/organoclay nanocomposites has been reported [25]. Based on this assumption, the secondary peak could be correlated to the XRD spectra of the PLA and PBS polymers itself in PLA/PBS/C30BX nanocomposites.

On the other hand, the WAXD profiles of nanocomposites of PLA/7, PBS/7 and PLA/PBS/7 are shown in Figure 1. The nanocomposite of $7 \mathrm{wt} \%$ of C30BX clay in PLA had an increased interlayer distance of $3.08 \mathrm{~nm}\left(2 \theta=2.86^{\circ}\right)$, while the PBS/C30BX (93/7 wt\%) nanocomposite had a clay interlayer distance of $3.06 \mathrm{~nm}\left(2 \theta=2.88^{\circ}\right)$, which indicated that PBS polymer also had a tendency to intercalate between C30BX layers. A better association or interaction between C30BX clay and PLA polymer could be concluded because of the larger interlayer distance of PLA/C30BX nanocomposite than that of PBS/C30BX nanocomposite. It was interesting to note further that when both PLA and PBS polymers were mixed with C30BX (PLA/PBS/C30BX: 80/20/7 wt\%), intercalation occurred. On the other hand, Wang et al. [26] studied the effect of clay in polypropylene (PP)/polystyrene (PS) blends, finding that when organophilic clay montmorillonite (OMMT) was mixed with either PP or PS, the layer distance of OMMT was around 3.2-3.3 nm. But, when both PP and PS were mixed with OMMT, the layer distance was increased further to $3.79 \mathrm{~nm}$. Thus, it was concluded that both immiscible polymers intercalated between the OMMT clay layers. Lim et al. [27] and Chen et al. [28] also confirmed the intercalation of immiscible polymer blend matrices within OMMT, which is in contrast to the results obtained in this study. It is noted that Chen et al. [28] also used a blend of PLA and PBS, but normal C30B, and they did not study the barrier properties.

It can also be found from Figure 1 that the addition of PBS to the PLA/C30BX (C30BX-7 wt\%) nanocomposite system shifted the basal peak towards the lower diffraction angles $(2 \theta)$ from $2.86^{\circ}$ to $2.2^{\circ}$ for PLA/PBS/C30BX (C30BX-7 wt \%). Intercalated peaks for both nanocomposites (PLA/C30BX and PBS/C30BX nanocomposites) were obtained at almost similar diffraction angles but the intensities were quite different. The characteristic peak height in PLA/C30BX nanocomposite was smaller as compared to PBS/C30BX nanocomposite. The low intensity of the peak can be explained by the tendency for delamination of C30BX in the PLA/C30BX mixture [29]. Whether C30BX has better affinity with PLA or PBS polymers can be determined by using the X-ray data of the ternary mixture. Figure 1 also showed that both PLA and PBS nanocomposites have peaks at the same diffraction angle; however, peak of PBS/C30BX nanocomposite is very sharp and has high intensity as compared to PLA/C30BX nanocomposite. This suggested that PLA chains have better affinity towards C30BX clay. Lee et al. [30] also reported similar results with biodegradable aliphatic polyester blends.

Meanwhile, our previously reported SEM image of the PLA/PBS blend of $80 / 20$ by wt $\%$ showed that the blend was immiscible [21]. Bhatia et al. [31] noticed a clear dispersed phase of PBS in all their compositions of PLA/PBS blends while investigating the compatibility between PLA and PBS polymers and concluded that for PBS contents higher than that in the $80 / 20$ (PLA/PBS) blends, that is, $20 \%$, the traditional morphology of immiscible blends could be seen. A similar type of immiscible morphology trend was reported for PLA and poly(butylene succinate adipate) blends by S. Lee and J. W. Lee [32]. On the other hand, TEM can further confirm the observations and the conclusions made from the WAXD data for the PLA/PBS/C30BX nanocomposites as previously reported [21]. Nonetheless for detailed analysis of gas barrier properties using Bharadwaj model, we need to reanalyze TEM images. Figure 2 shows TEM images of the PLA/PBS/C30BX nanocomposites with various clay loadings with the images chosen to show clay rich regions. Although the WAXD pattern of the nanocomposite having 1 and $3 \mathrm{wt} \%$ of C30BX showed featureless diffraction, which was interpreted as a delaminated morphology, in the TEM image (Figures 2(a) and 2(b)), delaminated/intercalated and stacked structure of the silicate layers could be readily observed for both 1 and 3\% clay loadings. More precisely, 1 and $3 \mathrm{wt} \%$ of C30BX exhibited a disordered intercalated structure of stacked silicates. Therefore, nanocomposites with 1 and $3 \mathrm{wt} \%$ of $\mathrm{C} 30 \mathrm{BX}$ are expected to achieve the greater enhancement in barrier properties. 

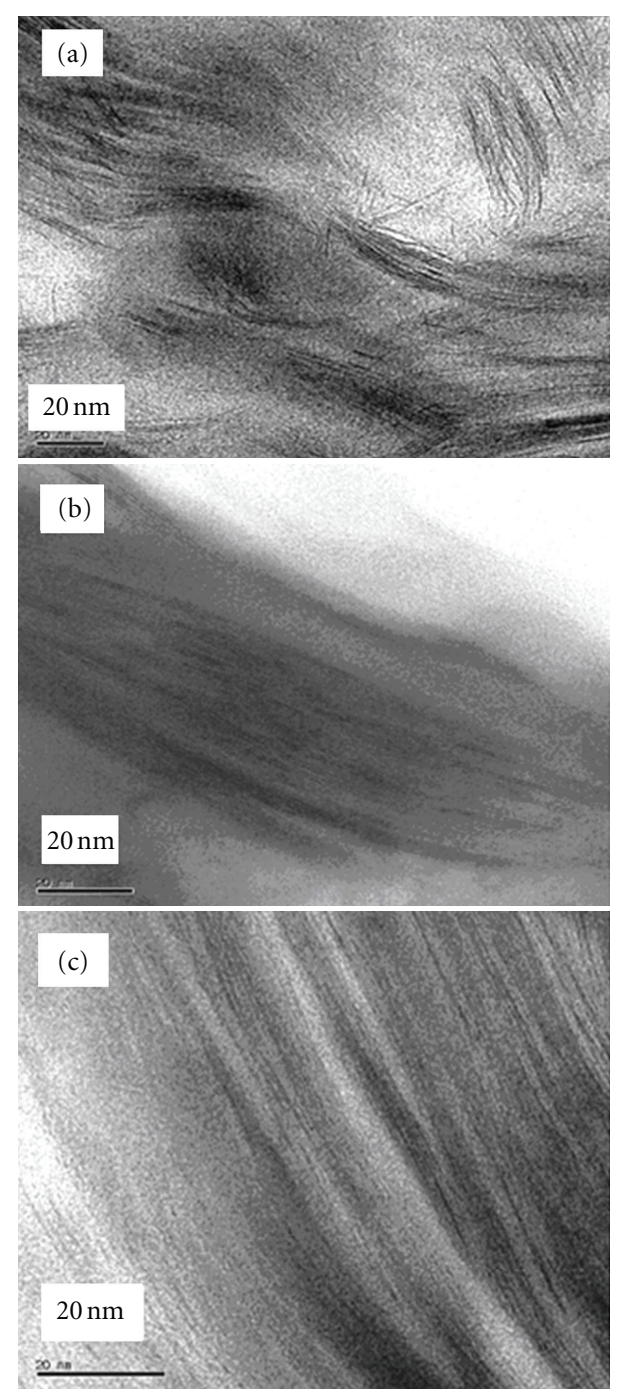

FIGURE 2: TEM images of PLA/PBS/Cloisite 30BX nanocomposites containing: (a) $1 \mathrm{wt} \%$, (b) $3 \mathrm{wt} \%$, and (c) $5 \mathrm{wt} \%$ clay. PLA/PBS ratio was $80 / 20$ by wt $\%$ for all the nanocomposites.

Big, clear, and flocculated silicate layers tactoids can be seen in the clay content $5 \mathrm{wt} \%$ (Figure 2(c)) and higher contents of nanocomposites as we previously observed [21]. It can be seen that with the increase of clay concentration, the thickness of the tactoids increased. However, these tactoids themselves gain some degree of disorderliness, which may be due to the clay concentration as well as the shearing forces during the melt extrusion process in the preparation of the nanocomposite. TEM images (not shown) clearly demonstrated that the stacked and intercalated silicate layers were nicely dispersed in the PLA and PBS matrices which were supported by WAXD analysis also. A previous section proved that both polymers were intercalated between the clay layers of C30BX (refer to Figure 1).

Sheet-like platelets (clay particles) are believed to increase the gas barrier properties in nanocomposites. The barrier property improves due to the tortuous path being created by the clay particles which retards the diffusion of the gas

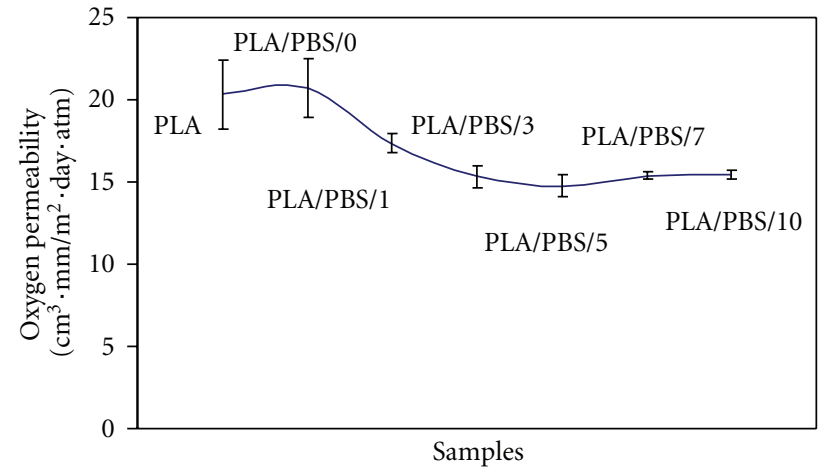

Figure 3: Effect of clay (C30BX) on oxygen permeability of PLA/PBS (80/20) blend and various PLA/PBS/C30BX (80/20/clay) nanocomposites of different clay loadings [23].

molecules through the matrix region. The theory behind the improvement in the gas barrier properties of the polymer layered nanocomposites was explained above.

All the nanocomposite films showed better oxygen barrier properties than neat PLA films as well as PLA/PBS blend films, as shown in Figure 3, which is previously observed [23]. Amongst all the nanocomposites, the nanocomposite having $5 \mathrm{wt} \%$ of $\mathrm{C} 30 \mathrm{BX}$ content exhibited the highest reduction in oxygen permeability, approximately $26 \%$. XRD and TEM (Figures $2(\mathrm{a}$ ) and $2(\mathrm{~b})$ ) results indicated that a mixture of exfoliated and disordered intercalated morphology occurred for 1 and $3 \mathrm{wt} \%$ of clay nanocomposites. However, improvement in $5 \mathrm{wt} \%$ of nanocomposite was the maximum. As the clay content was increased, aggregates of the clay particles formed which gave less improvement in oxygen barrier property. Research has shown that the degree of tortuosity also reflects geometrical influences like shape and state of delamination/intercalation of platelets and their orientation in the polymer matrix [12, 33-37]. For instance, Ray et al. [34] found a significant decrease in oxygen permeability for compression molded PLA nanocomposites samples, while observing some difference between the experimental value and calculated value due to the coexistence of intercalated stacked and disordered/delaminated clay layers. On the other hand, compression-molded specimens are generally known to give lower transmission rates than extruded specimens, while blown films generally show a good improvement in barrier properties [35], probably due to the fact that the biaxial orientation of silicate platelets in the polymer matrix produced during the blown film process would increase the tortuosity enough to enhance the barrier properties [36]. Therefore, the overall improvement in the barrier property was not that high because the specimens used in this study for obtaining oxygen permeability were compression molded. Nonetheless, it is interesting to note that, even though the oxygen permeability did not decrease further at higher clay contents (7 and $10 \mathrm{wt} \%$ ) in the PLA/PBS/C30BX nanocomposites, the decrease can be considered significant in comparison to the PLA/PBS (80/20) blend due to the presence of impermeable crystalline clay particles restricting the passage of oxygen. 


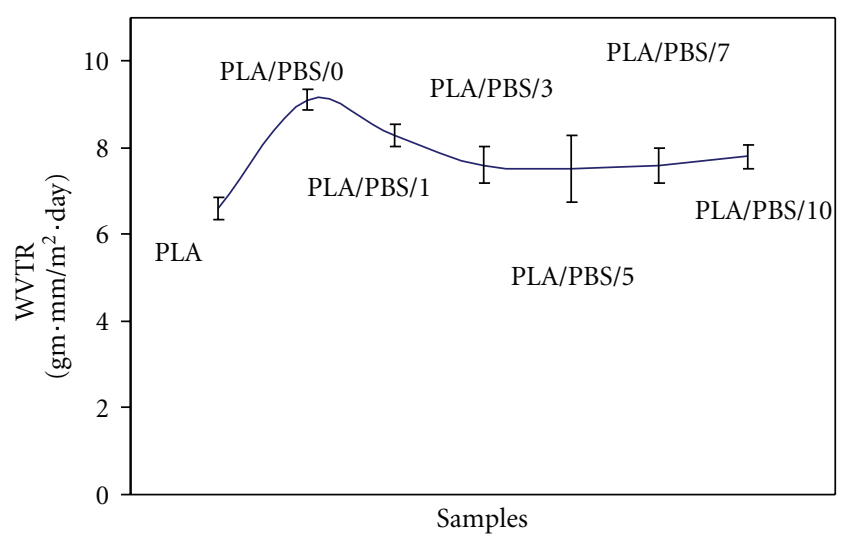

FIGURE 4: Water vapour transmission rates (WVTR) of PLA/PBS $(80 / 20)$ blend and their nanocomposites (PLA/PBS/C30BX) with various loadings of C30BX organoclay.

For the relatively modest improvement of the barrier properties in their compression molded nanocomposites, Krook et al. [35] explained two reasons of the void-content, which provided means for non-diffusion transport through the film and the nonuniformity of the clay particles. The improvement of the barrier properties of PLA/PBS/C30BX nanocomposites compared to PLA and PBS blend alone prepared by compression molding was probably because of the presence of the clay which served as barriers.

The PLA/PBS/C30BX nanocomposites also exhibited a modest improvement in water vapour barrier properties relative to those of the PLA/PBS blend films. Water vapour transmission rates (WVTR) of all the samples are shown in Figure 4. Nielsen [10] and Gerlowski [37] suggested a general principle for the transportation of water vapour. They stated that water penetrates the galleries between the montmorillonite layers, forming water clusters in the nanocomposites, and does not allow the water to pass through quickly, and thus the diffusivity and the overall transport of water across the films is decreased.

In the PLA/PBS/C30BX nanocomposites, the water vapour permeation rate was not reduced as much as in the case of the oxygen permeation rate due to the hydrophilic nature of the polymers. When the PBS was added to the PLA in the ratio of $80 / 20 \mathrm{wt} \%$ (PLA/PBS), the water vapour transmission rate increased. During the transport of water through the PLA/PBS films, water first absorbed through the one side of the film and once the saturation level was attained, the water started to diffuse through the film to the other side and transportation took place. The water vapour transmission rates decreased the most, up to $18 \%$, when only $5 \mathrm{wt} \%$ of C30BX was added in the binary blend. This decrease in water vapour transmission rate should be considered as quite modest, which implies that the C30BX also acted as impermeable layers which decreased the amount of tortuosity.

Note that the polymer crystalline regions in PLA and PBS polymers are considered to be impermeable to small molecules such as gases, which can lead to modifications in the final properties. It has been suggested that the presence of the filler (clay) may nucleate [38] crystallization, which leads to an increase in degree of crystallinity. This can have a direct effect on the transport properties. The significant drop in permeability with an increased crystallinity is usually explained in two ways [39, 40]. Firstly, inclusion of impermeable crystallites decreases the amount of amorphous material through which the permeant can diffuse. Secondly, impermeable clay crystallites increase the tortuosities of the transport path. This improvement in the gas barrier properties can be attributed to the achievement of the mixed morphology suggested by XRD and TEM results and a decrease in void density. Gas barrier properties were improved due to the distribution of clay in the polymer matrix. On the other hand, regarding the effect of clay on crystallization, it was observed from our previous study using DSC that while 1 and $3 \mathrm{wt} \%$ of clay present in PLA/PBS/clay nanocomposites may not be enough to notice effect on crystallinity, the crystallinity was the highest for $5 \mathrm{wt} \%$ of clay content [23].

As discussed earlier, according to the Nielsen model [10], the permeability of the nanocomposite $\left(P_{s}\right)$ is related to the permeability of the neat polymer $\left(P_{p}\right)$ and the volume fraction of the sheets $\left(\Phi_{s}\right)$, length $(L)$, and width $(W)$ of the sheets (clay particles), as given in (3), when $S$ is equal to 1 . The thickness of the basic structural unit of montmorillonite is well defined and is approximately given as $W \sim 1 \mathrm{~nm}$; however, the length and width show large distribution, ranging from $100 \mathrm{~nm}$ to several microns depending upon the concentration of the filler in the polymer. The effective width of the sheets of the nanocomposites can change through aggregation of clay layers. As seen from (2), the volume fractions of the sheets and the sheet length and sheet width are the required parameters for the calculations of relative permeability for nanocomposites.

Importantly, two main reasons made the estimation of sheet length quite complicated. Firstly, on the micrograph images of the nanocomposites, only the projections of the particle transect were seen. Therefore, the real dimensions could not be estimated. Secondly, the features appearing on the micrograph images were not straight; therefore, a computer assisted analysis method was required [41]. Unfortunately, a fully automated image analysis was excluded, because of the poor visibility of the particles on the image. However, a semiautomated image analysis was used to estimate the sheet length of the clay particles as given in Figure 5. The volume fractions of the sheets in the nanocomposites were calculated from the weight fraction using the density $\left(1980 \mathrm{~kg} / \mathrm{m}^{3}\right)$ of C30BX.

TEM images of polymers normally consist of different shades of gray colour which primarily indicate electron density contrast through the sample. Regions of high atomic number appear dark, while those of low atomic number appear light. The discrimination between the greyish polymeric regions and the dark lines corresponding to the alumino silicate platelets was relatively easy by eye. In our semiautomated image analysis method, conversion of the original TEM photomicrograph into a black and white image was necessary. 


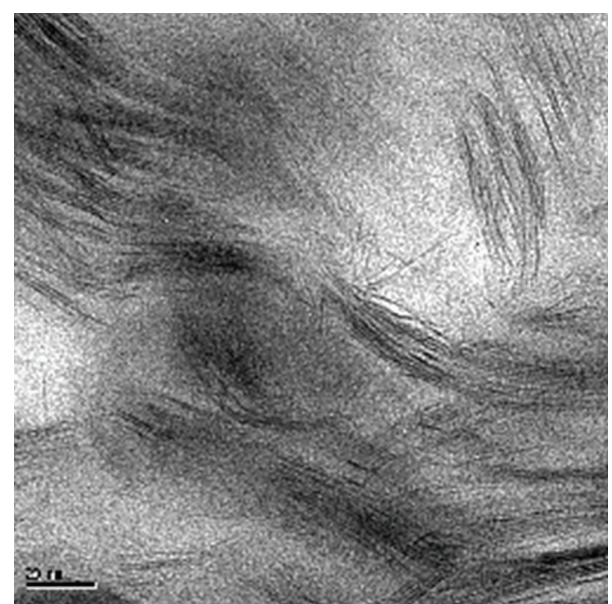

(a)

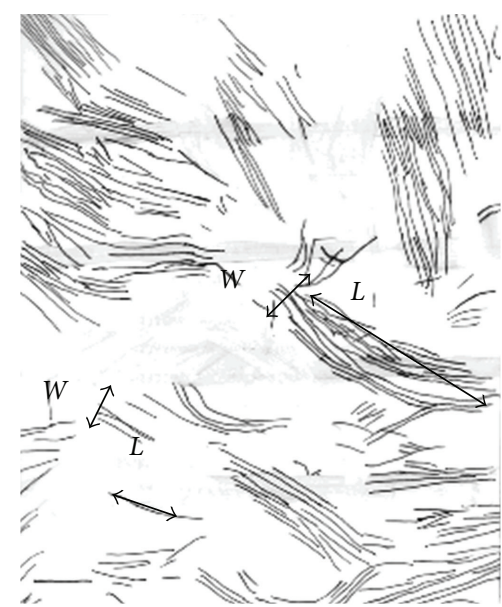

(b)

FIGURE 5: TEM (a) and its scanned image (b) of a transparent film obtained by manually tracing the particles by superimposing the film on the micrograph print. TEM image shown here is for PLA/PBS/C30BX nanocomposite containing $1 \mathrm{wt} \%$ of C30BX at $20 \mathrm{~nm}$.

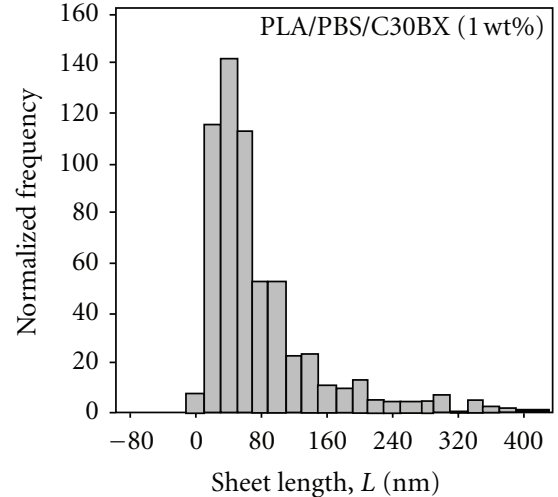

Average length Standard deviation

79.04 71.87

Total number of particles 603

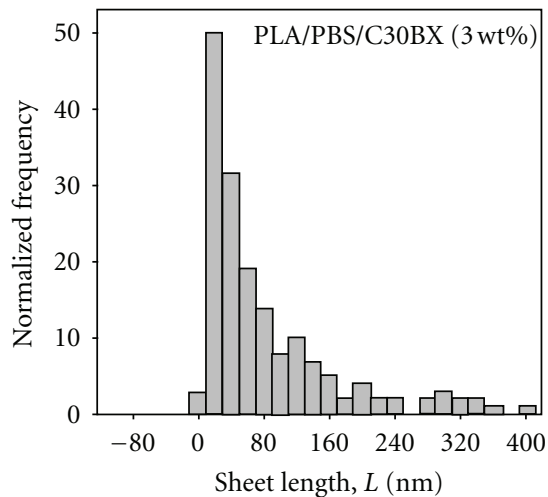
Average length
82.69
Standard deviation 83.64
Total number of particles 169

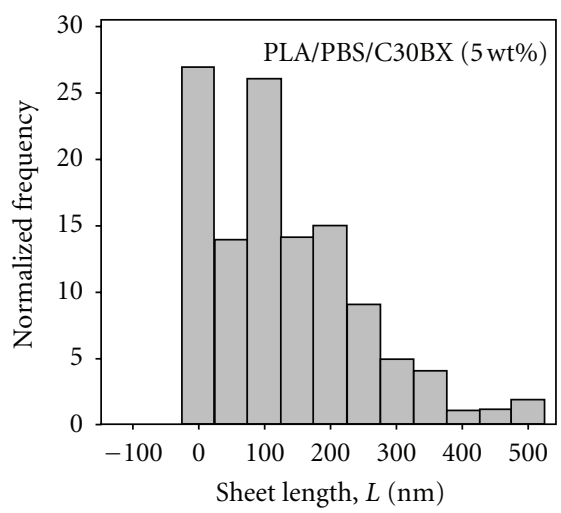

$\begin{array}{ll}\text { Average length } & 131.4 \\ \text { Standard deviation } & 114.5 \\ \text { Total number of particles } & 118\end{array}$
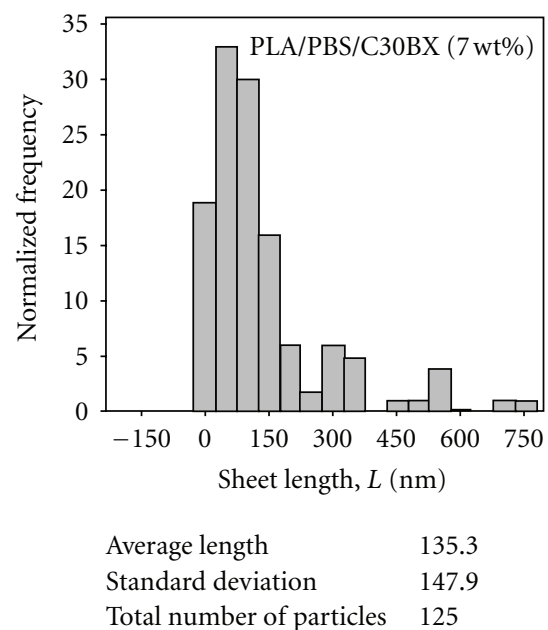
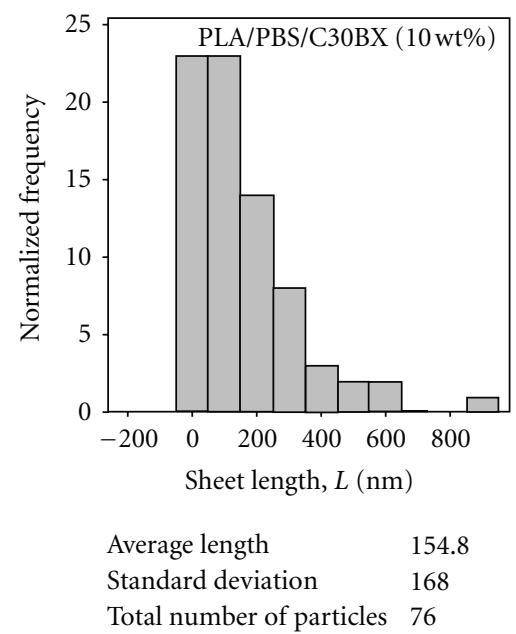

FIGURE 6: Particles length distribution for PLA/PBS/C30BX nanocomposites. Number in the bracket indicates loadings of C30BX. 


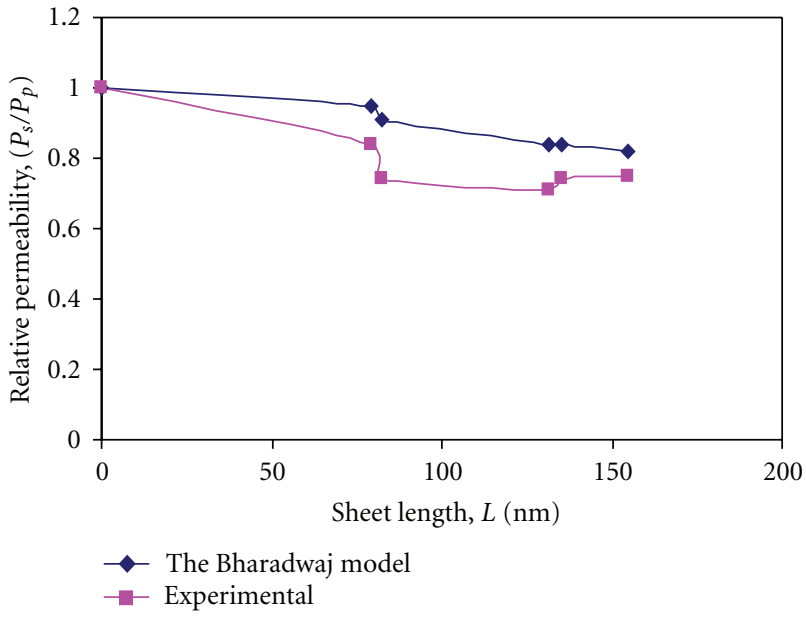

FIGURE 7: Relative permeabilities obtained from (1) and (2) taking exact values of effective width and experimental data (MOCON, oxygen permeability unit), showing the validation of the Bharadwaj model.

The semi-automated method [7] consisted of, first, manually tracing the dispersed platelets that were parallel to the beam and the agglomerates on a transparent film overlaid on the micrograph image print using a black permanent pen. The isolated platelets were considered as single platelets. The resulting transparent film was then electronically scanned and converted into a greyscale tagged-image film format (TIFF) image file. A micrograph image and its scanned image obtained by manually tracing the particles are shown in Figure 5. This greyscale image can then be converted into a black and white or binary image by using image analysis software (Scion Image Beta 4.02, Scion Corporation, USA). This software easily converts a TIFF file into a binary image and creates a duplicate of the image. From this file, the sheet lengths of the particles were calculated to obtain histograms for the various nanocomposites using Minitab 15 software.

Figure 6 shows histograms of the C30BX particle lengths and pertinent statistical data obtained on all the PLA/PBS/C30BX nanocomposites having various clay loadings. Here, the effects of clay concentration can be easily seen as sheet length increased with the addition of C30BX clay. Thus, average sheet lengths along with standard deviations were obtained. The calculated values of tortuosity and relative permeability of all PLA/PBS/C30BX nanocomposites using (2) are presented in Table 1. By comparing the relative permeabilities of oxygen obtained from the experiments and from the model, it can be seen that the experimental data very well validate the the Bharadwaj model for nanocomposites for the lower concentrations of C30BX. The nanocomposite of $1 \mathrm{wt} \%$ had the shortest sheet length and with the increase in clay loadings sheet length increased. At low concentrations of clay content, the clay particles were dispersed well as compared to the nanocomposites of higher content of C30BX (discussed earlier in XRD and TEM results). Therefore, lower concentration gave short clay lengths, while at higher concentrations, sheet length started to increase. From TEM images, it was seen that as concentration of clay increased more stacks of clay particles were formed. Therefore, the effective thickness of the clay sheets (tactoids) also increased. Instead of $1 \mathrm{~nm}$, it becomes 10 or $14 \mathrm{~nm}$ or more, depending upon the number of clay layers in the intercalated tactoids. However, here $S=1$ (planar arrangement of silicate layers to the path of gas molecule to diffuse) was taken as assumptions while doing the calculations using the Bharadwaj model based on the clay being a gas barrier. Due to the stacking of the clay layers, now the width of the clay particles becomes effective width with the value like $6,10,14,21$, and $33 \mathrm{~nm}$ approximately for the clay loading of $1,3,5,7$, and $10 \mathrm{wt} \%$. If these values are used in the Bharadwaj model while calculating the relative permeability, then curves for both the relative permeability of experimental and Bharadwaj model would match as shown in Figure 7.

Figure 7 shows that experimental data validated the Bharadwaj model up to a sheet length of approximately $80 \mathrm{~nm}$ (for 1 and $3 \mathrm{wt} \%$ ). After that, at high sheet lengths larger and larger deviations from the Bharadwaj model were observed. The reason behind this may be that certain assumptions, like good dispersion of clay particles in the polymer matrix, $S=1$ and $W \sim 1 \mathrm{~nm}$ were used in this model. Due to agglomeration/intercalation in PLA/PBS nanocomposites, the thickness of the sheet did not remain as $1 \mathrm{~nm}$. At high concentrations, thickness becomes effective thickness (effective width), which depends on the number of sheets in the stacks. The tendency of the silicate sheets to agglomerate was indeed expected to become more severe as the clay concentration increased. Due to the change in the thickness of the silicate stacks, its aspect ratio $(L / 2 W)$ also changed and the high enhancement in gas barrier was not noted. Thus, for high content of C30BX particles, the Bharadwaj model was not validated by the experimental data of PLA/PBS/C30BX nanocomposites.

If the actual values of width instead of $1 \mathrm{~nm}$ were used in (2), the relative permeability of PLA/PBS/C30BX nanocomposites would validate the Bharadwaj model for all the concentrations of C30BX.

\section{Conclusion}

PLA/PBS/Cloisite 30BX clay nanocomposites containing 1, 3 , 5,7 , and $10 \mathrm{wt} \%$ of clay were prepared by a melt extrusion method, and their morphology was examined using both $\mathrm{XRD}$ and TEM. Improvement in oxygen permeability was significantly noticed for PLA/PBS/C30BX nanocomposites with the addition of C30BX; however, the water vapour permeation rate showed only modest improvements in this system. In this study, compression molded samples were used for the determination of gas permeabilities. Due to the stacks of clay and non-uniform dispersion of clay particles, tortuosity path for the gas molecules did not increase sufficiently to make a significant improvement in gas barrier properties. PLA/PBS/C30BX nanocomposites validated Bharadwaj model for only up to $3 \mathrm{wt} \%$ of clay content. For higher concentrations of C30BX, deviation from 
TABLE 1: Permeability parameters used to validate the Bharadwaj model. Number in the bracket indicates loadings of C30BX.

\begin{tabular}{|c|c|c|c|c|}
\hline \multirow[b]{2}{*}{ Samples } & \multirow{2}{*}{$\begin{array}{c}\text { Volume fraction, } \Phi_{s} \\
\text { Sheet length, } L(\mathrm{~nm}) \\
\text { Width of the sheet, } W(\mathrm{~nm})\end{array}$} & \multirow[b]{2}{*}{ Tortuosity factor, $\tau$} & \multicolumn{2}{|c|}{ Relative permeability } \\
\hline & & & Model & Experimental \\
\hline \multirow{3}{*}{ PLA/PBS/C30BX (0 wt\%) } & 0 & & & \\
\hline & 0 & 1 & 1 & 1 \\
\hline & 0 & & & \\
\hline \multirow{3}{*}{ PLA/PBS/C30BX (1 wt\%) } & 0.0065 & & & \\
\hline & 79.04 & 1.25 & 0.95 & 0.84 \\
\hline & 6 & & & \\
\hline \multirow{3}{*}{ PLA/PBS/C30BX (3 wt\%) } & 0.0187 & & & \\
\hline & 82.69 & 1.77 & 0.91 & 0.74 \\
\hline & 10 & & & \\
\hline \multirow{3}{*}{ PLA/PBS/C30BX (5 wt\%) } & 0.0314 & & & \\
\hline & 131.4 & 3.06 & 0.84 & 0.71 \\
\hline & 14 & & & \\
\hline \multirow{3}{*}{ PLA/PBS/C30BX (7 wt\%) } & 0.0443 & & & \\
\hline & 135.3 & 3.99 & 0.84 & 0.74 \\
\hline & 21 & & & \\
\hline \multirow{3}{*}{ PLA/PBS/C30BX (10 wt\%) } & 0.0625 & & & \\
\hline & 154.8 & 5.83 & 0.82 & 0.75 \\
\hline & 33 & & & \\
\hline
\end{tabular}

the Bharadwaj model was observed due to the presence of clusters and agglomerates.

\section{Acknowledgment}

The authors would like to thank Mr. Hwang Sun Jae for his valuable assistance with the transmission electron microscopy (TEM).

\section{References}

[1] S. S. Ray and M. Okamoto, "Polymer/layered silicate nanocomposites: a review from preparation to processing," Progress in Polymer Science, vol. 28, no. 11, pp. 1539-1641, 2003.

[2] D. Garlotta, "A literature review of poly(lactic acid)," Journal of Polymers and the Environment, vol. 9, no. 2, pp. 63-84, 2001.

[3] T. Fujimaki, "Processability and properties of aliphatic polyesters, "BIONOLLE", synthesized by polycondensation reaction," Polymer Degradation and Stability, vol. 59, no. 1-3, pp. 209-214, 1998.

[4] N. S. Oliveira, J. Oliveira, T. Gomes, A. Ferreira, J. Dorgan, and I. M. Marrucho, "Gas sorption in poly(lactic acid) and packaging materials," Fluid Phase Equilibria, vol. 222-223, pp. 317-324, 2004.

[5] K. Bahari, H. Mitomo, T. Enjoji, F. Yoshii, and K. Makuuchi, "Radiation crosslinked poly(butylene succinate) foam and its biodegradation," Polymer Degradation and Stability, vol. 62, no. 3, pp. 551-557, 1998.

[6] Y. Doi, "Biodegradable plastics and polymer," Journal of Pesticide Science, vol. 19, no. 1, pp. S11-S14, 1994.

[7] T. D. Fornes and D. R. Paul, "Modeling properties of nylon 6/clay nanocomposites using composite theories," Polymer, vol. 44, no. 17, pp. 4993-5013, 2003.
[8] S. S. Ray, K. Yamada, M. Okamoto, A. Ogami, and K. Ueda, "New polylactide/layered silicate nanocomposites. 3. Highperformance biodegradable materials," Chemistry of Materials, vol. 15, no. 7, pp. 1456-1465, 2003.

[9] S. S. Ray, K. Okamoto, and M. Okamoto, "Structure-property relationship in biodegradable poly(butylene succinate)/ layered silicate nanocomposites," Macromolecules, vol. 36, no. 7, pp. 2355-2367, 2003.

[10] L. E. Nielsen, "Models for the permeability of filled polymers systems," Journal of Macromolecular Science, Part A, vol. 1, no. 5, pp. 929-942, 1967.

[11] A. S. Michaels and R. B. Parker, "Sorption and flow of gases in polyethylene," Journal of Polymer Science, vol. 41, no. 138, pp. 53-71, 1959.

[12] R. K. Bharadwaj, "Modeling the barrier properties of polymerlayered silicate nanocomposites," Macromolecules, vol. 34, no. 26, pp. 9189-9192, 2001.

[13] K. Yano, A. Usuki, A. Okada, T. Kurauchi, and O. Kamigaito, "Synthesis and properties of polymide-clay hybrid," Journal of Polymer Science, Part A, vol. 31, no. 10, pp. 2493-2498, 1993.

[14] E. P. Giannelis, R. Krishnamoorti, and E. Manias, "Polymersilicate nanocomposites: model systems for confined polymers and polymer brushes," Advances in Polymer Science, vol. 138, pp. 107-147, 1999.

[15] P. C. Lebaron, Z. Wang, and T. J. Pinnavaia, "Polymer-layered silicate nanocomposites: an overview," Applied Clay Science, vol. 15, no. 1-2, pp. 11-29, 1999.

[16] K. Yano, A. Usuki, and A. Okada, "Synthesis and properties of polyimide-clay hybrid films," Journal of Polymer Science, Part A, vol. 35, no. 11, pp. 2289-2294, 1997.

[17] E. P. Giannelis, "Polymer layered silicate nanocomposites," Advanced Materials, vol. 8, no. 1, pp. 29-35, 1996.

[18] K. E. Strawhecker and E. Manias, "Structure and properties of poly(vinyl alcohol)/ $\mathrm{Na}^{+}$montmorillonite nanocomposites," Chemistry of Materials, vol. 12, no. 10, pp. 2943-2949, 2000. 
[19] P. B. Messersmith and E. P. Giannelis, "Synthesis and barrier properties of poly(epsilon-caprolactone)-layered silicate nanocomposites," Journal of Polymer Science Part A, vol. 33, pp. 1047-1057, 1995.

[20] D. Cava, A. L. Rubio, L. Cabedo et al., "Comparative performance and barrier properties of biodegradable thermoplastics and nanobiocomposites versus PET for food packaging applications," in Proceedings of the Society of Plastics Engineers Annual Technical Conference (ANTEC'05), pp. 83-86, May 2005.

[21] A. Bhatia, R. K. Gupta, S. N. Bhattacharya, and H. J. Choi, "An investigation of melt rheology and thermal stability of poly(lactic acid)/poly(butylene succinate) nanocomposites," Journal of Applied Polymer Science, vol. 114, no. 5, pp. 28372847, 2009.

[22] S. W. Ko, R. K. Gupta, S. N. Bhattacharya, and H. J. Choi, "Rheology and physical characteristics of synthetic biodegradable aliphatic polymer blends dispersed with MWNTs," Macromolecular Materials and Engineering, vol. 295, no. 4, pp. 320$328,2010$.

[23] A. Bhatia, R. K. Gupta, S. N. Bhattacharya, and H. J. Choi, "Effect of clay on thermal, mechanical and gas barrier properties of biodegradable poly(lactic acid)/poly(butylene succinate) (PLA/PBS) nanocomposites," International Polymer Processing, vol. 25, no. 1, pp. 5-14, 2009.

[24] V. Krikorian and D. J. Pochan, "Poly (L-lactic acid)/layered silicate nanocomposite: fabrication, characterization, and properties," Chemistry of Materials, vol. 15, no. 22, pp. 43174324, 2003.

[25] J. H. Chang, Y. U. An, and G. S. Sur, "Poly(lactic acid) nanocomposites with various organoclays. I. Thermomechanical properties, morphology, and gas permeability," Journal of Polymer Science Part B, vol. 41, no. 1, pp. 94-103, 2002.

[26] Y. Wang, Q. Zhang, and Q. Fu, "Compatibilization of immiscible poly(propylene)/polystyrene blends using clay," Macromolecular Rapid Communications, vol. 24, no. 3, pp. 231235, 2003.

[27] S. K. Lim, J. W. Kim, I. Chin, Y. K. Kwon, and H. J. Choi, "Preparation and interaction characteristics of organically modified montmorillonite nanocomposite with miscible polymer blend of poly(ethylene oxide) and poly(methyl methacrylate)," Chemistry of Materials, vol. 14, no. 5, pp. 1989-1994, 2002.

[28] G. X. Chen, H. S. Kim, E. S. Kim, and J. S. Yoon, "Compatibilization-like effect of reactive organoclay on the poly(1lactide)/poly(butylene succinate) blends," Polymer, vol. 46, no. 25, pp. 11829-11836, 2005.

[29] G. Chen, X. Chen, Z. Lin, W. Ye, and K. Yao, "Preparation and properties of PMMA/clay nanocomposite," Journal of Materials Science Letters, vol. 18, no. 21, pp. 1761-1763, 1999.

[30] C. H. Lee, H. B. Kim, S. T. Lim, H. J. Choi, and M. S. Jhon, "Biodegradable aliphatic polyester-poly (epichlorohydrin) blend/organoclay nanocomposites; synthesis and rheological characterization," Journal of Materials Science, vol. 40, no. 15, pp. 3981-3985, 2005.

[31] A. Bhatia, R. K. Gupta, S. N. Bhattacharya, and H. J. Choi, "Compatibility of biodegradable poly (lactic acid) (PLA) and poly (butylene succinate) (PBS) blends for packaging application," Korea Australia Rheology Journal, vol. 19, no. 3, pp. 125-131, 2007.

[32] S. Lee and J. W. Lee, "Characterization and processing of biodegradable polymer blends of poly(lactic acid) with poly(butylene succinate adipate)," Korea Australia Rheology Journal, vol. 17, no. 2, pp. 71-77, 2005.
[33] T. C. Bissot, Barrier Polymers and Structures, ACS Symposium Series, American Chemical Society, Washington, DC, USA, 1989, edited by W. J. Koros.

[34] S. S. Ray, K. Yamada, A. Ogami, M. Okamoto, and K. Ueda, "New polylactide/layered silicate nanocomposite: nanoscale control over multiple properties," Macromolecular Rapid Communications, vol. 23, no. 16, pp. 943-947, 2002.

[35] M. Krook, A. C. Albertsson, U. W. Gedde, and M. S. Hedenqvist, "Barrier and mechanical properties of montmorillonite/polyesteramide nanocomposites," Polymer Engineering and Science, vol. 42, no. 6, pp. 1238-1246, 2002.

[36] C. Thellen, C. Orroth, D. Froio et al., "Influence of montmorillonite layered silicate on plasticized poly(l-lactide) blown films," Polymer, vol. 46, no. 25, pp. 11716-11727, 2005.

[37] L. Gerlowski, Barrier Polymers andStructures, ACS Symposium Series, American Chemical Society, Washington DC, USA, 1989, edited by W. J. Koros.

[38] D. J. Sekelik, E. V. Stepanov, S. Nazarenko, D. Schiraldi, A. Hiltner, and E. Baer, "Oxygen barrier properties of crystallized and talc-filled poly(ethylene terephthalate)," Journal of Polymer Science Part B, vol. 37, no. 8, pp. 847-857, 1999.

[39] W. R. Vieth, Diffusion in and Through Polymers, Hanser, Munich, Germany, 1991.

[40] M. Hedenqvist and U. W. Gedde, "Diffusion of small-molecule penetrants in semicrystalline polymers," Progress in Polymer Science, vol. 21, no. 2, pp. 299-333, 1996.

[41] M. Vincent, Characterisation of exfoliation and intercalation in polymer/layered silicate nanocomposites [M.S. thesis], Department of Chemical Engineering, McGill University, Montreal, Canada, 2004. 

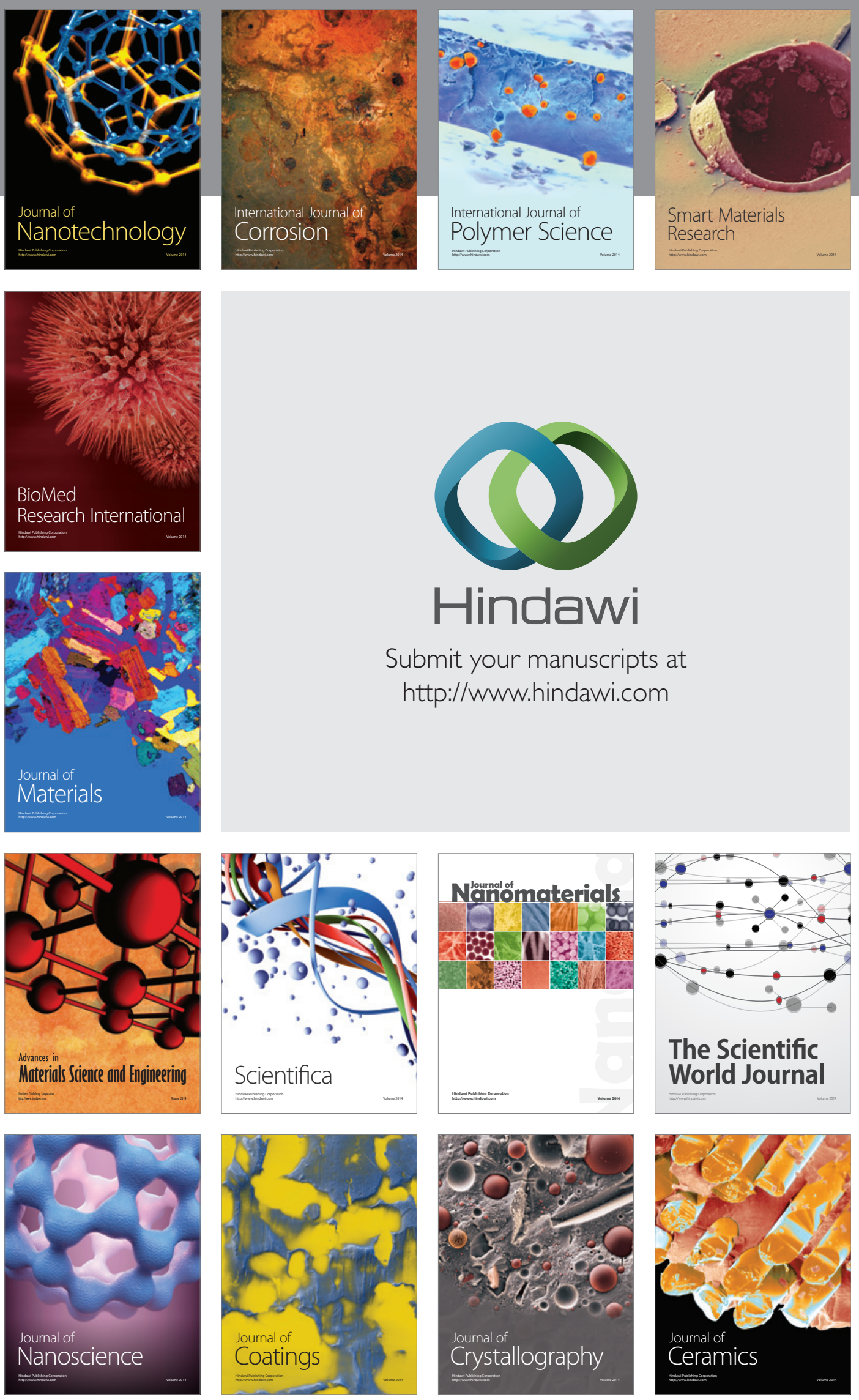

The Scientific World Journal

Submit your manuscripts at

http://www.hindawi.com

\section{World Journal}

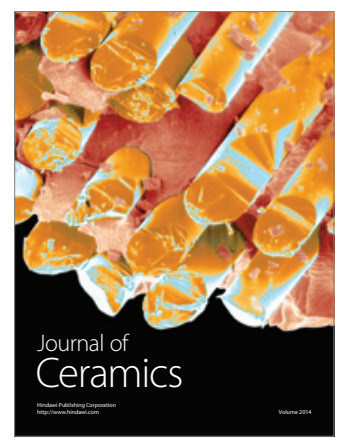

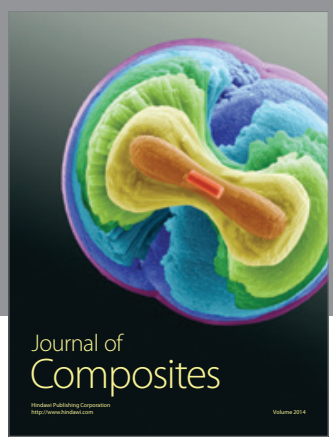
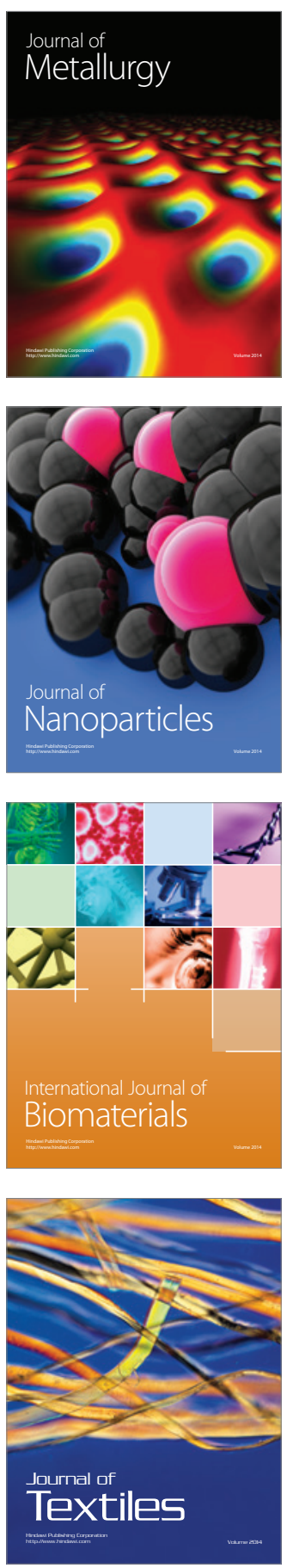